\title{
Obtaining Reliability Insights during a Product's Conceptual Design Process through Bayesian Network Modeling
}

\author{
Luis Mejia Sanchez, Cummins Inc and Rong Pan*
}

School of Computing, Informatics, and Decision Systems Engineering, Arizona State University, Arizona, USA

\begin{abstract}
The philosophy of Build-In-Reliability (BIR) or Design for Reliability (DFR) emphasizes the value of reliability prediction at a product's conceptual design stage. Due to the lack of reliability data, reliability assessment of a new design is not usually performed at this stage. In this paper, we propose a methodology to provide the reliability insight of a new design concept. The methodology consists of three major processes: functional analysis, cognitive map and Bayesian network modeling. A case study is given to demonstrate our proposed method.
\end{abstract}

Keywords: Product's conceptual design; Bayesian network

\section{Introduction}

Accurate early reliability prediction becomes a common requirement for new product's development as systems have grown to be more complex $[1,2]$. However, in the design phase of a new product there are not physical samples to assess or prove reliability. On the other hand, under the philosophical influence of Design For Reliability (DFR) or Build-In- Reliability (BIR), significant efforts had been put on reliability improvement by product design. For example, the use of computer support analysis (i.e., computer simulation) by designers is widely spread [3]. Also, as complements to these computer simulation tools, qualitative and quantitative information from similar existing designs are also important to BIR [4]. In recent years with the aid of new computational technologies, several design approaches have been proposed with the use of Bayesian reliability. Bayesian methods for system reliability analysis have been studied extensively in the work by Hamada et al., Wang et al., Pan and Rigdon [5-7]. These publications have depicted the possibility of assessing a new product's reliability before a physical sample is viable by taking into consideration of all existing information. Such information may include component and subsystem data, information from similar existing systems, and expert's opinions. Nowadays, there is also broad literature considering the reliability information integration aspects. Johnson et al., Hamada et al. and Wilson et al. [8-10] proposed a fully hierarchical Bayesian method for reliability assessment of multi-component system. They studied the multilevel data scenario with pass/fail, lifetime or degradation data [11]. Further extensions to these works include Anderson-Cook et al., Graves, Hamada and Reese et al. [12-14] whose focus were on binomial data or lifetime data under a known failure structure situation. Johnson et al. [15] described a hierarchical Bayesian model for assessing the early reliability of a complex system.

However, the previous studies of reliability information integration had overlooked the product design process. A product design process consists of four main phases: planning and task clarification, conceptual design, embodiment design and detailed design [16]. The activities of planning and task clarification result in the specification of information that contains the requirements and constraints the new product should embrace. Conceptual design refers to the analysis and identification of design concepts and the construction of functional structures for new products that meet the accorded requirements. The embodiment design phase occurs when a detailed structure is defined and the corresponding physical structures (prototypes) are created for further validation. Finally, in the detailed design phase, design improvements are implemented, manufacturability is reviewed, and production is scheduled. As suggested in the literature, most DFR techniques were implemented at the embodiment or detailed design stage, but there are few that explicitly addressed it during the conceptual design stage. In the work of Huang and Jin [17], they reduced the "gap" between reliability requirements and conceptual design by using stress and strength interference theory. Also, Derelov [18] provided a qualitative model for investigating potential failure modes in the conceptual design phase. Stone et al. [19] used the Function-Failure Design Method (FFDM) to assess reliability in early design phases. This research was extended by Kurtoglu and Tumer [20], where they presented a functionfailure identification and propagation process through hierarchically modeling system functions in the conceptual design phase. Similarly, Grantham Lough et al., Krus and Grantham Lough [21] combined the functional model of a design with the historical knowledge base of specific functions, along with their risks, to provide a quantitative assessment of function-based failure propagation.

In the conceptual design phase, detailed physical information is not produced, as there is no physical part to test; thus, all the classic reliability methods cannot be used. Furthermore, traditional methodologies operate under the assumption that there is a failure structure that can be derived by reliability tools. However, deriving a new product's reliability structure (e.g., reliability block diagram or fault tree) is also a challenging task in the conceptual design phase. Nevertheless, it is desired to have a reliability insight during this phase as it can guide the decision making process for the new product development. For example, the early knowledge of product reliability may drive its reliability improvement plan, improve the test planning process, and ultimately affect the product's warranty plan and its total life-cycle cost. Hence, in order to assess reliability in the conceptual design stage, a non-classical approach is needed.

${ }^{*}$ Corresponding author: Rong Pan, School of Computing, Informatics, and Decision Systems Engineering, Arizona State University, Arizona, USA, Tel: (480) 965-4259; E-mail: rong.pan@asu.edu

Received August 11, 2017; Accepted August 28, 2017; Published September 11, 2017

Citation: Sanchez LM, Inc C, Pan R (2017) Obtaining Reliability Insights during a Product's Conceptual Design Process through Bayesian Network Modeling. Ind Eng Manage 6: 224. doi:10.4172/2169-0316.1000224

Copyright: (c) 2017 Sanchez LM, et al. This is an open-access article distributed under the terms of the Creative Commons Attribution License, which permits unrestricted use, distribution, and reproduction in any medium, provided the original author and source are credited. 
This research addresses the challenges in reliability assessment at a product's conceptual design stage. Our investigation starts from the idea of integrating information from similar proven concepts (parents) into a new product's conceptual design. In order to achieve this, a coherent and novel system reliability structure revelation process is proposed. First, it calls for the study of product's functional structures via a cognitive map. Then, the cognitive map is converted to a quantifiable Bayesian network by using parenting analysis and expert opinion elicitation. Finally, once the Bayesian network model is completed, the designer or engineer can assess and validate the new product's reliability requirement.

The remainder of this paper is organized as follows. In Section 2, the basics of functional analysis, cognitive maps and Bayesian network are introduced. Section 3 discusses the proposed methodology, where the integration of the parent information and eliciting information modeled a reliability structure for a new product in the conceptual design phase. For a better understanding of this proposed methodology, a case study is exhibited in Section 4. Lastly, in Section 5 a discussion is held and future work is proposed.

\section{Background and Framework}

\section{Conceptual design}

As an early phase in the engineering design process, conceptual design can be summarized as the creation of functions and their combinations that satisfy an established need. There are different approaches to conceptual design, as they can be developed for specific products. However, the most common approach is the one defined by Pahl et al. [16]. Additionally, Huang and Jin [17] described the typical tasks based on different approaches. In a general sense, the steps included in the conceptual design phase are stated in Table 1. Also, Table 1 shows reliability considerations that should be made in each steps.

However, the consideration of reliability in these steps does not provide an assessment of product reliability at this point, but merely specify the reliability requirements for the new design. Hence, a more systematic approach is needed in order to ensure reliability in the conceptual design phase.

\section{Functional analysis}

Various definitions of product function can be found in literature [16,22-25]. In general, a product function is defined as the relationship between input and output to satisfy a need or requirement. Function analysis is a systematic process that identifies all functions of a system as well as the relationships and interactions between them and their elements (sub-functions). Its main ob- jective is to reduce product complexity by dividing the principal characteristic of the system into manageable functions. A primary (overall) function can be decomposed to several sub-functions, and the decomposition can be performed on several levels as necessary. For more information of functional analysis, please refer to Pahl et al., Otto, Wood and Stone and Wood [16,26,27]. In general, these methodologies can be summarized by two steps: (1) identify all elements involved and (2) depict their relationships. These relationships are usually graphically depicted (i.e., matrices or graphs). Therefore, a common end result of functional analysis is known as the functional structure. As a consequence, a functional structure provides not only the structure be- tween sub-function and its elements but also the potential interactions among them. This becomes critical to reliability assessment when there is no actual physical system.

Figure 1 shows the transition between functionalities of the conceptual design selected to the breakdown of these functions. The graphic representation of subfunctions is known as the functional structure. Therefore, a system might have several functional structures, with each for one of its functionalities. Figure 2 graphically shows the assumed functional structures for each system function. Let Si denote the functional structure set for system function $\mathrm{i}$, which contains $\mathrm{s}_{1 i}$, $\mathrm{s}_{2 i}, \ldots, \mathrm{s}_{m i}$ subfunctions, then the total system functionality for the new product can be represented by

$$
\bigcup S_{i} \text { for } i=1,2, \ldots, n \text {. }
$$

An increased use of functional analysis in reliability assessment has been seen in the literation. Stone et al., Tumer, Stone, Bryant et al., Kurtoglu, Tumer, Grantham Lough et al. and Krus and Grantham Lough [28-30] discussed the importance of functional analysis for reliability prediction in the conceptual design phase. Their research focused on the function to failure design method, which promotes early identification of potential failures by linking them to product functions. This approach consists of defining the relationship between system functions and its failure modes in a matrix form, then historical data, expert inputs, as well as standardized design taxonomies, are utilized to define those relationships. It is a qualitative approach to the recognition of potential functional failures before a concept is selected.

In this research the function to failure approach is used. Furthermore, the use of functional analysis is to set a baseline to reveal reliability insights in the conceptual design stage. To identify relationships between functions, a graphical structure is created through a cognitive map [31] to depict the risk for failures in functions, a parenting process is chosen to assess failure rates. Moreover, inside the parenting process there is a branch called elicitation process [32], where experts' opinions on one or more uncertain quantities are synthesized [33]. Hence, the elicitation process is used to gain the desired insight into the reliability of new product. This process will be explained in the next section.

\section{Conceptual Design Process Steps}

1. Abstractly identify essential requirements against design criteria

2. Establish functional structures

3. Search and combine solution principles to satisfy the requirements.

4. Select suitable candidates for concept variants

\section{Reliability Considerations}

New product's main function is formulated. Reliability requirements must be set in this step as they will aid in identifying which ones are essential functions of the new design.

When creating functional structures there are three considerations that are recommended: (1) Logical consideration (2) Physical considerations and (3) Reliability considerations. For reliability the functional structures must take into account those reliability requirements defined in the previous step.

Reliability requirements must be present when looking into different solution alternatives/combinations that will fulfill the functional structures previously defined. This can be done using conventional methods or bias/unbiased related approaches for the searching and systematic (logical) or/and using mathematical models for combining (Pahl et al., 2007).

In order to start evaluating the possible solution they must meet different criteria. The criteria might include: manufacturability, safety, maintainability and reliability

5. Evaluate technical and economic feasibility for Reliability constraints must carry a high weight into the selection and optimization process. concept variants. 


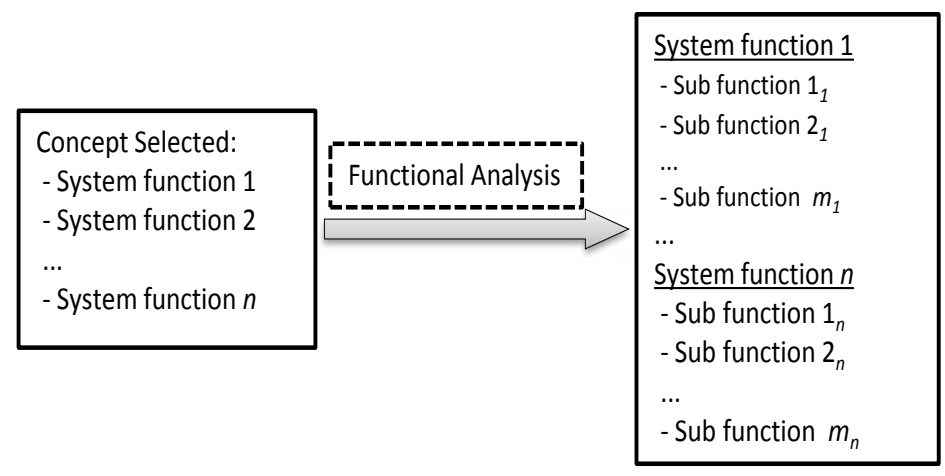

Figure 1: Functional analysis for the new product in the conceptual design phase.
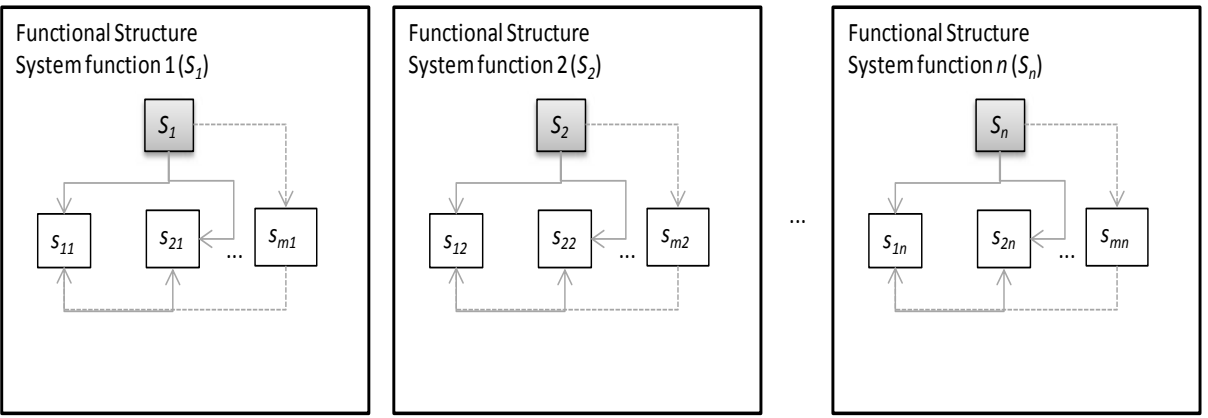

Figure 2: Functional structures for the system functions of new product.

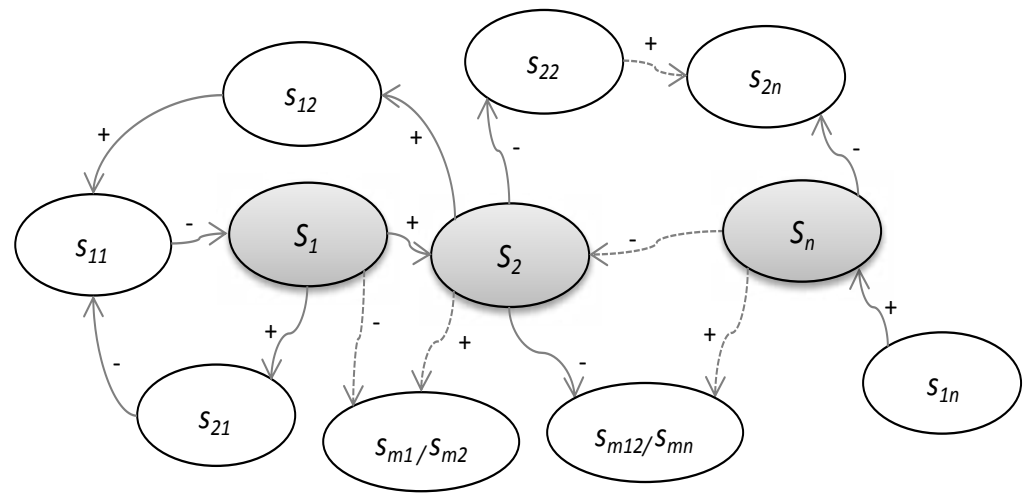

Figure 3: High level cognitive map for new product's functions/requirements.

\section{Cognitive maps}

Cognitive map (CM) is essentially a graphical representation of the knowledge or the perception of a given system. It can be defined as a signed digraph where nodes represent concept variables and directed arcs are the causal relationships [31]. Tolman [34] first introduced the $\mathrm{CM}$ concept and it was defined as a visual representation of an influence network between concepts. Since then, CMs have been applied in several different fields including medical, psychology, software and engineering among others. Therefore, nowadays there exist a vast collection of definitions and methodologies in the literature [35-37]

To illustrate the process, Figure 3 presents one of their general uses of a cognitive map for a given system.
The "+" or "-" sign indicates either positive or negative correlation between the conceptual functions, respectively. As observed, this type of maps just graphically represents qualitative information for causality but does not allow for any kind of quantitative computation.

In order to offer the quantification capability in CM, fuzzy cognitive maps (FCM) has been introduced in the literature [38,39]. In a FCM a weight is used to depict the strength of causalities, along with a numerical value that is assigned to each node, which would express its state or level. Then, FCMs are simulated in discrete or continuous time while the weights remain constant, but the state/level values change. During the simulation, a premeditated threshold function is used to update the weighted sum of all values that are inputs to a node. 
However, the simulation may not produce an answer to the design problem. Other disadvantages of FCM include the use of thresholds that need to be pre-defined and, as other fuzzy systems, this method is incapable of self-learning when a new evidence is collected [40]. Nevertheless, CM provides an excellent graphical representation of conceptual relationships among functions. In this research, cognitive maps are used for graphing the reliability-wise relationships of functions, thus, allowing a better understanding of the functional behavior that lead to system failure.

\section{Bayesian networks}

Bayesian networks (BNs), also called belief networks, are used to represent knowledge about an uncertain domain [41]. To be more specific, a BN represents a set of Bayesian random variables and their conditional dependencies using a directed acyclic graph (DAG). In the graph, each node represents a random variable, while the arcs/edges between the nodes represent the probabilistic dependencies among the corresponding random variables. These conditional dependencies in the graph are often estimated by using known statistical and computational methods.

The use of BNs in reliability has proved to have significant advantages over traditional approaches [42]. One of these advantages over Reliability Block Diagrams (RBD) and Fault Tree Analysis (FTA), resides in the use of the probabilistic relationships. For example, given the deterministic nature of the gates for a FTA it is difficult to incorporate the uncertainty seen in the conceptual phase of the design. Conditional probabilities in a $\mathrm{BN}$ allows to capture this uncertainty between functional relationships. Furthermore, BN also provides the opportunity of combining different sources of information (i.e., expert's input) to present an overall assessment of a system.

In a mathematical sense $\mathrm{BN}$ is defined as a compact representation of a multivariate statistical distribution function. Then, its graphical model encodes the set of conditional independence statements. This grants the possibility of calculating the joint probability function as:

$$
f\left(x_{1}, \ldots, x_{n}\right)=\prod^{n} f\left(x_{i} \mid \operatorname{pre}\left(x_{i}\right)\right),
$$

Where pre(xi) represents the predecessor nodes of variable $\mathrm{xi}$, hence $\mathrm{f}(x i \mid \operatorname{pre}(x i))$ is defined as the conditional probability function for variable node xi given its predecessors.

Furthermore, BNs have two different sets of information. The qualitative part of the model is represented by the DAG structure, which, for this study, is defined by the CM and functional analysis. Secondly, the quantitative aspect is provided by the parameters of the model. These parameters are needed for conditional probability functions, where the probabilistic dependencies between a node and its predecessor node are specified. The values of these parameters can be determined by using the knowledge from parent products or from expert opinions. Figure 4a presents a basic BN structure, which includes the probabilities that would form the joint distribution of all nodes. Figure $4 \mathrm{~b}$ shows how the probability tables can be represented in a matrix form.

Inferences in BNs are made by queries and they can be classified by (1) the causal inference, which can be seen graphically as a top-down approach (from failure cause to failure mode), and (2) the evidential inference, which is a bottom-up approach (from the observation of a variable to infer the state of a different variable). In the product design framework, queries are made based on what designers need to evaluate. There are simply queries such as the posterior marginal distribution that might be used to assess the reliability of a concept. Moreover, there also exist conditional queries that help designers to make decisions or to provide the information of features of a design. Additionally, sensitivity analysis can be implemented to investigate if the design specfifications that meet the proposed requirements. The algorithms for solving these queries can be exact inference algorithms, such as enumeration, belief propagation (polytrees), variable elimination or Clustering/Joint tree algorithms, or approximate inference algorithms, such as stochastic simulation / sampling methods, Markov chain Monte Carlo methods, genetic algorithms, neural networks, simulated annealing or mean field theory. For more information please refer to Bishop et al. [43].

The goal for this research is to gain reliability insights from conceptual designs. BNs can provide these insights because it models the causal relationships between functions in a system. Using BNs to represent the system reliability/failure structure can capture the explicit and implicit interactions between individual design functions, and thus various inference procedures can be performed to further decipher the

a)

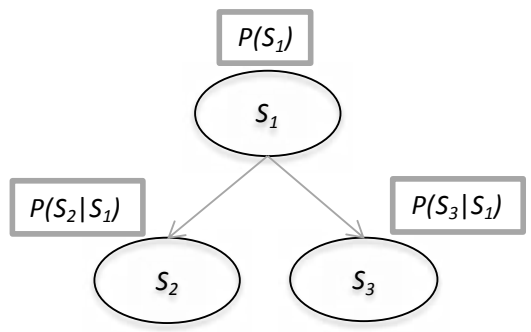

b)

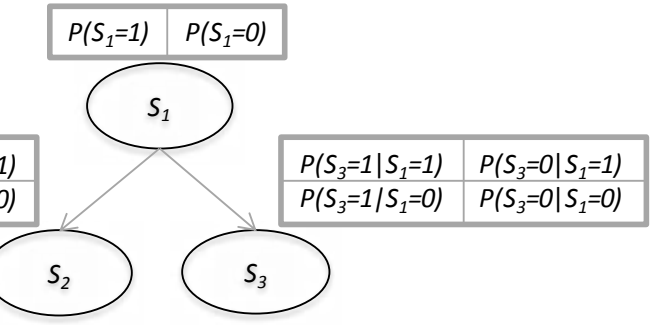

Figure 4: Bayesian Network representations a) BN with conditional probability function b) BN with conditional probability table with binary variables 
conceptual system. The methodology proposed to achieve this goal is described in the next section.

\section{Methodology}

In order to assess a new product's reliability, it is necessary to take into consideration many product- specific factors such as product definition, design purpose, the level of change from previous designs, etc. In other words, the analysis methods and the tools to be used should be determined on the case-by-case basis. However, our proposed methodology presents a general approach to gaining reliability insights regardless of these factors. The general framework is depicted on Figure 5. It shows the progression from one phase to another and the tools to link them.

\section{Concept and functions}

The methodology starts in the conceptual design phase, when a concept has been selected. Since there are not physical design representations at this time, the requirements are translated to functionalities of the new product. Therefore, either new functions or already established ones are identified and/or defined as the outcome of this phase.

\section{Function to failure structures}

Once the system functions are defined a functional analysis needs to be conducted. The first step consists in the identification of the primary or main function(s) and all the sub-functions involved. Secondly, the relationships between them need to be depicted. It is recommended to use a graphical representation when performing both steps to define the functional structures.

In order to have a reliability structure (or failure structure) in the early design process it is important to identify failure modes even when physical components have just been conceptualized. In this instance, using the function to failure approach [44] creates the possibility to define a failure when a function is not executed as expected.

However, given the uncertainty in conceptual design, assessing the failure probability for all the functions can be challenging. On the other hand, those functions can be identified and related to different existing products or parents. Moreover, additional functional information can be obtained by other sources. For example, simulation, expert opinions, early experimentation, literature, etc. Then, parent information might be defined as the existing available information coming from current design/products that have similar functions or sub-functions of the new design/product.

\section{Functional structures to cognitive map}

Following the methodology depicted by Augustine et al. [31] it was possible to obtain a cognitive map model from functional structures. The procedure incorporates in a stepwise manner, all structural, functional, and causal aspects of the system. Cognitive Maps Fragments (CMF) are formed for the each one of the system functions identified. After the CMFs are finalized, they can be automatically aggregated into the final cognitive map (CM) structure by using the simple union operation expressed in Equation 3:

$$
C M=\left(\bigcup_{m=1}^{i} N_{m}\right) \bigcup\left(\bigcup_{m=1}^{i} A_{m}\right)
$$

Where, $\mathrm{N} m$ represent the set of $i$ nodes and Am the $j$ arcs from the CMFs.

The construction of the cognitive map should be taken with expert inputs. In the creation of the cognitive map redundant sub-functions (i.e., sub-functions that are shared by more than one system function) are depicted as such and it also reduces the complexity of the graph. Then, the relationships between each one of the functions were explicitly stated given the arcs in the map. Moreover, when creating the CMFs there is the possibility to capture additional functions to depict interactions between failure modes that were not capture by the functional analysis.

\section{Cognitive map to Bayesian network}

A general functional structure has been already defined by the Cognitive Map (CM); however, the qualitative relationships between functions in CM will not provide an objective form to gain the desired reliability insight. A more objective approach to integrate information, and moreover, to continuously integrating further information updates, is to use a Bayesian network (BN). $\mathrm{BN}$ is a tool that aggregates the impacts of changes on components/sub-functions to the system/main function level and allocates the total risk to different sub-functions (i.e., identify sub-functions with high failure risk).

A few publications have discussed the similarities and differences between CM and BN. Nadkarni, Shenoy, Nadkarni and Shenoy $[45,46]$

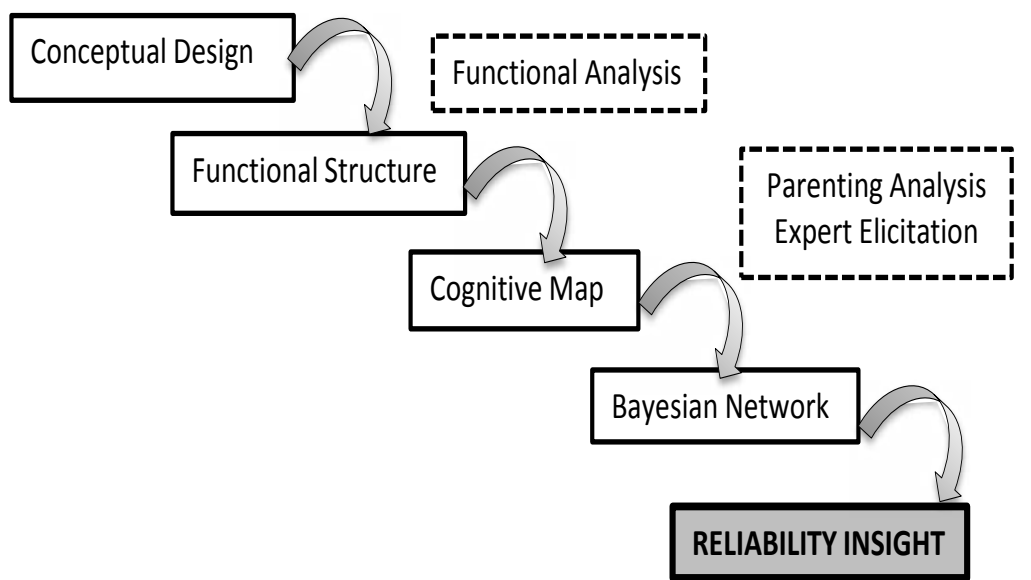

Figure 5: Methodology framework to gain reliability insight on the Design phase for a new product. 
pointed out the main differences (or biases) between $\mathrm{CM}$ and $\mathrm{BM}$ as follows:

- Conditional Independence. In CM, arcs between variables depict dependence; however the absence of an arc does not imply independence. On the other hand, the lack of an arc among variables in a BN it does implies conditional independence among them.

- $\quad$ Cause-Effect relations. This bias refers to the perception of the effect coming from causes or if the relationship is depicted from effects to causes. It is important to establish a deductive relationship (causes to effects) which is the proper way to have a CM converted into a BN. Furthermore, it is recommended to be cautious with adductive relationships (causes from effects).

- Direct vs. Indirect relationship. Differentiation between direct and indirect cause-effect arcs permits the incorporation of conditional independencies in CM. Thus, this facilitates the translation to a $\mathrm{BN}$.

- Circular relations. They exits in CM as subjective judgments are made and also they might represent time changing relations between variables. However, they violate the acyclic graph-ical structure for $\mathrm{BN}$. Hence, it is required to eliminate circular relations to make CM compatible with BN.

Moreover, in their research they presented a 4-step procedure to construct Bayesian Cognitive Maps (BCM). These steps are (1) Expert elicitation, (2) Derivation of CM, (3) Modification to CM to create BCM, and (4) Derivation of the parameters of BCM. Step (1) and (2) are defined by a structured interview with domain experts and by coding their answers into a cause-effect map. Step (3) is focused on making the $\mathrm{CM}$ compatible with the $\mathrm{BCM}$ by considering the four biases presented above along with expert elicitation. This step is critical to the successful implementation of BCM. Nadkarni and Shenoy [46] provided the following checklist for constructing a valid $\mathrm{BN}$ :

- It is important to ensure that the lack of links between concepts in a causal map implies conditional independence and the presence of links between concepts implies dependence.

- Reason the underlying cause-effect relations between concepts. Causal statements involving abductive reasoning could be misrepresented in a causal map by an arc from effect to cause, then such misrepresentations should be corrected.

- Distinguish between direct and indirect relationships. A link between two concepts in a causal map may imply a relation that can be either direct or indirect; while in $\mathrm{BN}$, only direct relations (between parent nodes and child nodes) should be depicted.

\section{- $\quad$ Eliminate any circular relations.}

Finally, in Step (4) a BN quantification process is implemented in two steps identifying the state space of nodes and deriving conditional probabilities. Unfortunately, Nadkarni and Shenoy [45,46] did not provide the details of how to perform parameterization of BN. Once these parameters are given, probability propagation (i.e., Bayesian belief propagation) algorithms might be used to make inferences. An example of this process is presented in Aktas et al. [47] where they use this approach to improve the efficiency of resource allocation in a health care facility.

In this research, the proposed methodology already covers steps (1) and (2) by going from the functional analysis in conceptual design to the CM. Step (3) was the generalization of graphically converting a CM to a BN. For step (4) parenting processes would be used.

Parenting process provides an objective data analysis process for transferring $\mathrm{CM}$ to $\mathrm{BN}$. The general guidelines are provided in Mejia Sanchez and Pan [48]. This approach would be especially helpful in generating Conditional Probabilities Tables (CPTs) for BNs. There are two main approaches to obtaining the parameters values - expert elicitation and synthesis of failure information from parent products. Following the guidelines for eliciting probabilities [33] and parenting process, the expert would be asked to provide an assessment of the marginal conditional probability inside the CPT. On the other hand, if there are existing products (parents) performing similar functions under the same conditions, their failure information can be translated or used directly into the CPT.

Consider a single function and two direct causes (other functions) that govern the successful execution of this function. Then, given two states to each cause and the CPT as shown in Table 2, there are four parameters, $p 1, p 2, p 3, p 4$, that need to be specified. Although it is uncommon in practice, we start our discussion with this naive scenario a complete historical dataset (log file) of the states of the function and its direct causes is available. This is possible if this function and its associated causes are continuously monitored by sensors and the log data from existing products that perform the same function can be obtained.

Using all observed instances of function states and cause states, it is straightforward to obtain the estimation of the conditional failure probability given a combination of cause states. For the previous example,

$$
p_{i}=P\left(F=1 \mid C 1_{i}, C 2_{i}\right)=\frac{\sum_{k} I_{k}\left(F=1, C 1_{i} C 2_{i}\right)}{\sum_{k} I_{k}\left(C 1_{i}, C 2_{i}\right)}
$$

Where the denominator is the total number of instances of the specific combination of $\mathrm{C} 1$ and $\mathrm{C} 2$ and the numerator is the number of instances of function failure at this combination.

However, even this simple formula could become troublesome in practice when there are many states for each cause node. In such case, the number of combinations grows large, thus the log file could be highly fragmented. Additionally, there might be no observation for a particular combination. Therefore, it is better to combine Equation (4) with the expert's opinion on how many function failure may happen for a given parent nodes combination. This is equivalent to assign a prior distribution to the function failure probability. Assume a Beta prior distribution, beta( $\mathrm{a} i, \mathrm{~b} i)$, for $\mathrm{p} i$, then the posterior estimation of $\mathrm{p} i$ is given by:

$$
p_{i}=\frac{\sum_{k} I_{k}\left(F=1, C 1_{i} C 2_{i}\right)+a_{i}}{\sum_{k} I_{k}\left(C 1_{i}, C 2_{i}\right)+\left(a_{i}+b_{i}\right)},
$$

Where $a_{i}+b_{i}$ is the equivalent sample size in the prior and $a_{i}$ is the equivalent number of failures in prior samples.

Therefore, in the expert opinion elicitation process, two questions would be asked: In your experience, how frequent this type of

\begin{tabular}{|c|c|c|c|}
\hline$C 1$ & $C 2$ & $P(F=1 \mid C 1, C 2)$ & $P(F=0 \mid C 1, C 2)$ \\
\hline 0 & 0 & $p 1$ & $1-p 1$ \\
\hline 0 & 1 & $p 2$ & $1-p 2$ \\
\hline 1 & 0 & $p 3$ & $1-p 3$ \\
\hline 1 & 1 & $p 4$ & $1-p 4$ \\
\hline
\end{tabular}

Table 2: Conditional probability table for a single function and two direct causes. 


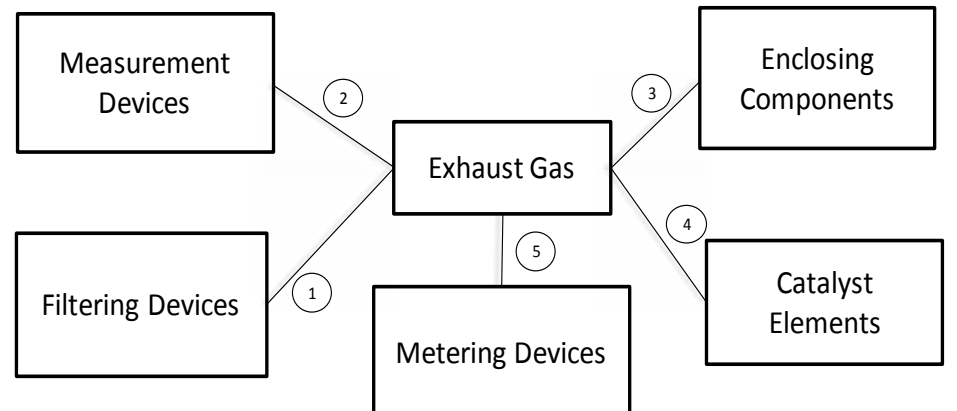

b)

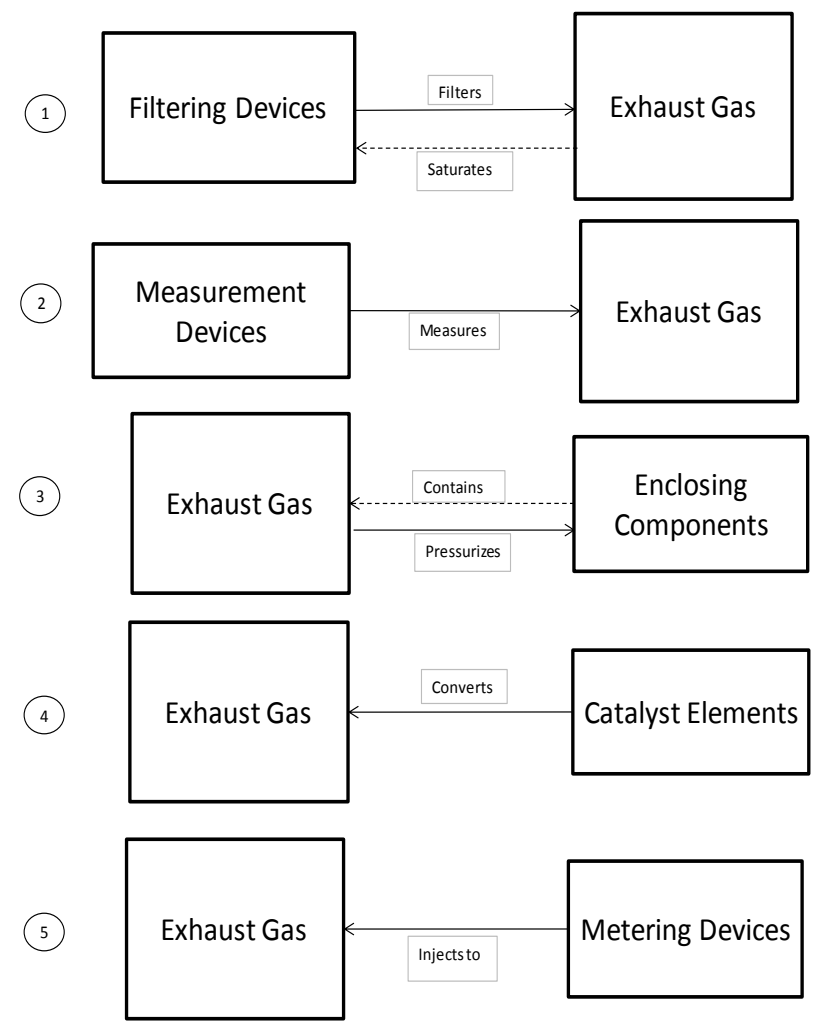

Figure 6: Functional analysis for the new CRD where a) presents the system functions and subfunctions, b) the system's functional structures.

combination of $\mathrm{C} 1$ and $\mathrm{C} 2$ may happen? And, in your experience, what is the chance of function failure given this type of combination of $\mathrm{C} 1$ and $\mathrm{C} 2$ ? The prior parameters, $\mathrm{a}_{\mathrm{i}}$ and $\mathrm{b}$, can be derived from the answers of these questions. By combining expert assessments and historical data, a robust conditional failure probability table can be obtained.

It is also common that the complete log files of parent products are not available; instead, one may have a record of failure frequency and failure causes of parent products. Yontay et al. [48] described a mathematical approach to obtain conditional probabilities from this type of failure records.

Aside from parenting and expert elicitation, meta-analysis is another possible approach to estimate probabilities when information is scarce. Meta-analysis refers to methods that focus on contrasting and combining results from different studies, in the hope of identifying patterns among study results, sources of disagreement among those results or other interesting relationships that may come to light in the context of multiple studies. This approach is usually more timeconsuming than other approaches.

\section{Bayesian network inference and evaluation}

The quantitative part of the $\mathrm{BN}$ is constructed after obtaining the conditional probability values. Therefore, it can be used now to make inferences about the functions in the model. The scope of this research is to have a graphical insight into the reliability of a new product in its conceptual design phase by obtaining a BN. The subsequent steps of how to use BN depend on each specific case, e.g., concept evaluation, assessment of unobservable parameters or conduct a sensitivity analysis. If concept evaluation is needed, researchers could use the joint probability distribution from the different concepts and proceed with a decision making process. Marginal conditional distribution and conditional dependencies can be used to estimate variables or parameters that were not observed. This can be achieved through the joint distribution of BN, i.e., probabilistic inference [49]. Lastly, evidential inference (or evidence propagation) refers to the ability to 
obtain marginal probabilities of parameters of interest, conditional on arbitrary configurations of other parameters based on the observed evidence [50].

There are several commercial software available for the inference and analysis of BN, such as Hugin (www.hugin.com) and Netica (www. norsys.com). There are also some development tools, such as MSBNx (research.microsoft.com/msbnx) and SamIam (reasoning.cs.ucla.edu/ samiam), that automate the process of inference based on existing algorithms [51]. These tools allow users to enter the BN structure graphically, input the observable details, and then do inference of either type (i.e., top-down or bottom-up).

\section{A Case Study}

In order to better demonstrate and validate the proposed methodology, a case study is introduced in this section to explore and clarify the concepts presented in the methodology section. This case study was derived from a real engineering design process in a major heavy equipment manufacturing company in U.S. Note that to avoid disclosing sensitive information, the values presented in this case study were masked and certain variables were removed. The parameter values given in the graphical model were elicited from domain experts by following the guidelines provided in Cooke [33], Mejia Sanchez and Pan [32].

\section{A contaminant reduction device (CRD)}

An automotive industry was developing a contaminant reduction device (CRD) to launch in the upcoming years. Since the Environmental Protection Agency (EPA) had restricted the emission levels on the years ahead, a new CRD was needed to comply with the new regulations. Additionally, a corporate reliability target at the end of product warranty was set to comply with a specific percentage of sales based on the marketing research. To comply with the regulation and to achieve the reliability targets, the design team proposed several improvements on their CRDs currently in production. Hence, the requirements for the new CRD were laid out and the design engineering team described the level of change needed in order to meet them.

A CRD is a device used to convert exhaust emissions, usually toxic, into less-toxic substances. The main objective of CRD is to stimulate a chemical reaction through the exhaust flow and additives in which contaminants are reduced. The design development of the new CRD was in the conceptual design phase; hence, to maximize resources and minimize further costs, the reliability team was tasked to assess the product's reliability at this early stage. Since data for the new model was scarce, the reliability team implemented the methodology described in this paper to create a graphical model to depict and integrate all information available. Moreover, using the company's reliability database experts were able to pull the information of parent products to make a better assessment on the new concept.

A concept was already selected based on the predetermined requirements, customer expectations and targets. Therefore, it was relatively easy to list the different functions that the new CRD was going to perform. Once system functions were identified, then a graphical structure was needed. The team performed a functional analysis where the basic functional structure was defined. Figure 6 shows the results of this exercise. Function analysis, as described in Section 2, can be performed using different approaches. The selected approach would depend on the level of expertise of the team and information and resources available. In the end, all results would yield in a main function and the relationship with its subfunctions represented graphically.

Following the rules established on Augustine et al. [32] for function taxonomy, a follow- up exercise was conducted to name the functions that were going to be used in the next step of reliability assessment. This exercise, was conducted to validate the functional structures by experts' consensus using parents' functional structures. For example, "[G] flow OF exhaust gas" was considered the main function of the system and it is expressed by its main subfunctions that form the failure functional structure in Figure 6a. The final list of the subfunctions, derived from the functional structures shown in Figure 6b, are given below. For example, "injection OF fluids" shows (5) and (2), therefore this subfunction comes from the functional structure number 5 and 2 .

Next, the list of functions from the functional structures needed to be represented in a graphical display. A CM was used to organize the different functional structures and to establish the causal relationships between all conceptual functionalities including subfunctions that might be repeated on different functional structures. Figure 7 presents the final $\mathrm{CM}$ obtained either by combining the individual CMFs

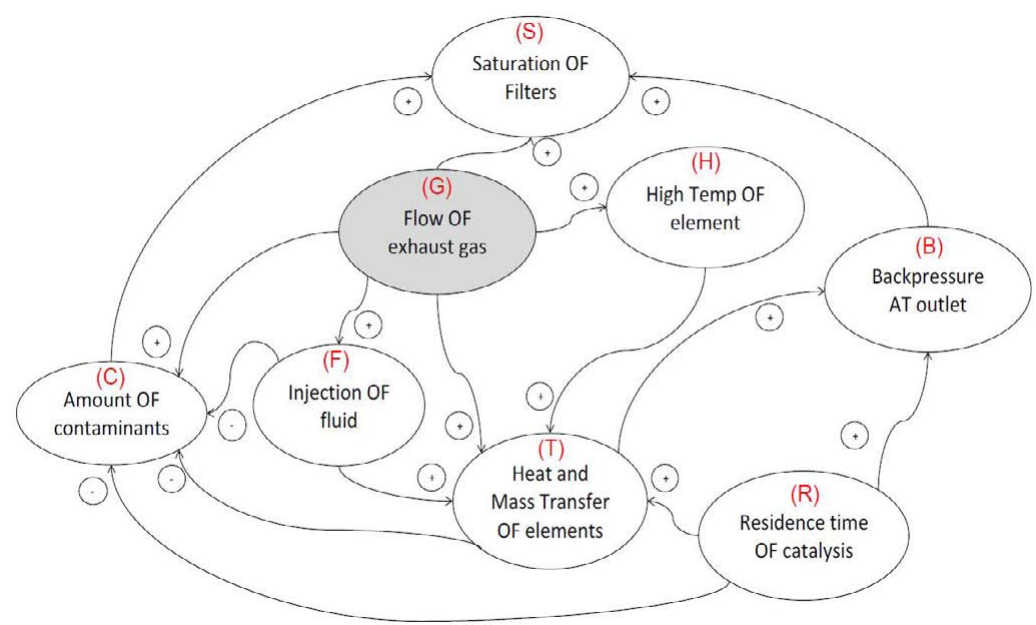

[S] Saturation OF filters (1); [C] Amount of contaminants (2) (1); [B] Backpressure AT outlet (3); $[H]$ High temperature OF elements (2) (4) (3); $[F]$ Injection OF fluids (5) (2); [R] Residence time OF catalysis (4) (2); [T ] Heat and mass transfer OF elements (2) (4).

Figure 7: Cognitive Map given functional structures for the system functions of new product. 
as discussed in Augustine et al. [32], dictating for highly complex structures, or as in this case establishing the relationships directly in a final map.

Following the proposed methodology, the obtained CM needs to be converted into a $\mathrm{BN}$. An extensive session was held to receive feedback from experts within the design team. Then, the conversion of the $\mathrm{CM}$ into a $\mathrm{BN}$ was executed following the recommendations made by Nadkarni and Shenoy mentioned in Section 3. Here, similarly to the functional analysis, the resultant $\mathrm{BN}$ would depend on the information and resources available as well as the experience of the team performing the task. However, it is important to remark that the current BN presents a mere window into the reliability insight of the product under design. Later when more product reliability data become available, using the Bayesian information updating mechanism this network can be easily updated.

Figure 8 represents the qualitative part of the BN. The next step is to obtain the conditional probabilities for each node. As this study is set in the early design stage, the design team decided to choose only binary states of each node function failure or nonfunctional (1) and functioning properly or functional (0). One of the reasons for using binary variables is that it aids the simplification of a complex problem, which is particularly valuable in the early design stage. Nevertheless, the power of $\mathrm{BN}$ resides in its properties, whether it is managing nodes with multiple states and/or performing analysis with continuous nodes. However, the number of conditional probabilities to be elicited could quickly grow to unmanageable if there are many states of many nodes needed to be specified. The scalability problem of this $\mathrm{BN}$ approach in early product design will be investigated in our future study.

Once the BN variables are defined, a parenting session was held to properly assess the required conditional probabilities. In this session, the company's reliability databases, the part-returns systems, as well as initial performance testing on available technologies were used by the team of experts in order to assess different function failure probabilities. Finally, experts' opinions were elicited to obtain the CPTs. The details of each function parenting process are described below:

- $\quad$ Node [Flow OF exhaust gas (G)]. As main function it did not required to change on is general functionality. Hence, CPT was obtained directly from current (parent products) function's failure information.

- Node [High Temperature OF element (H)]. Previous catalytic element had the latest tech- nology available and there is no plan to change if it meets the proposed requirements. In consequence, in a functional level its CPT would be also obtained by using the current function failure information.

- $\quad$ Node [Injection OF fluid (F)]. After an elicitation process for this particular function, it was determined that the metering devices would need to improve in order to meet new standards. In this elicitation session the CPT was computed by comparing different application where $\mathrm{F}$ function had performed in similar conditions.

- Node [Residence time OF catalysis (R)]. The time for the chemical process to take place has great variability. Hence, an elicitation



Figure 8: Bayesian network given functional structures from cognitive map. 
process was required to have a better understanding of the function. A CPT was provided based on parent information, analytical studies performed and expert input. The resultant CPT is shown in Figure 9.

- Node [Heat/Mass Transfer OF elements (T)]. The CPT for this function was obtained by a similar process of the enhanced parenting process from Mejia Sanchez and Pan [33]. The translation is simple when $\mathrm{H}, \mathrm{F}$ and $\mathrm{R}$ are seen as the failure causes for each state $(0$ or 1) and in consequence state of $\mathrm{T}$ represents the failure mode.

- $\quad$ Node [Amount OF contaminants (C)]. As new standards are imposed regarding the amount of allowable quantity of contaminants out of the system; new measure devices are needed to verify that this function is performing adequately. In order to obtain more objective estimates, different sensor groups were elicited and a consensus was reach on its CPT depicted in Figure 9.

- $\quad$ Node [Backpressure AT outlet (B)]. One of the biggest requirements was to overhaul the enclosing components. It was required to change in size and form. Therefore, this function was one of the main concerns. After several sessions of elicitation, experts were able to evaluate the CPT given that the characteristic of this functionality were seen in a different application.

- $\quad$ Node [Saturation OF Filters (S)]. Functionality of the filtering devices did not suffer major changes as they would be required to operate under the same conditions. Hence, direct parenting provided the CPT for this function.

For better understanding of the team, all information was compiled in Figure 9. It is important to remind the reader that most values presented here are masked or altered given confidentially agreements. Moreover, as noted in the description of the process, each elicitation (or parenting analysis) is unique, therefore the integration of information for each node has its own procedure.

The CPTs complete the quantitative part of the BNs. Consequently, this $\mathrm{BN}$ model can provide insights to the reliability of this new design, depending on the queries made to the BN. For the new CRD, the design team was looking at the three different scenarios to be discussed in the next three sections.

\section{Sensitivity analysis}

The main need to have a reliability insight is to verify that the functions for the chosen CRD concept would meet the requirements established from the different environmental regulations and customer expectations.

On the CRD's BN, all functions' CPTs in Figure 8 were able to be elicited or parented. Hence, at this point both teams (design and reliability) were interested to see if the current concept was capable to meet specific requirements to measure emission compliance standards by $90 \%$, and more importantly, which function parameters needed to be improved in order to meet the specification.

Figure 10 shows the CRD's BN displayed by SamIam. The parameters were estimated based on the CPTs from Figure 9. It was observed that the "amount OF contaminants" (C) function was only

\begin{tabular}{|c|c|c|c|c|c|}
\hline \multirow{2}{*}{$\begin{array}{l}\text { Flow OF exhaust Gas } \\
\text { (G) }\end{array}$} & Nonfunctional & $P(G=1)=0.074$ & & & \\
\hline & Functional & $P(G=0)=0.926$ & & & \\
\hline \multicolumn{2}{|c|}{ Flow OF exhaust gas } & Nonfunctional & Functional & & \\
\hline \multirow{2}{*}{$\begin{array}{c}\text { High Temperature OF } \\
\text { elements }(\mathrm{H})\end{array}$} & Nonfunctional & $P(H=1 \mid G=1)=0.714$ & $P(H=1 \mid G=0)=0.044$ & & \\
\hline & Functional & $\mathrm{P}(\mathrm{H}=0 \mid \mathrm{G}=1)=0.286$ & $\mathrm{P}(\mathrm{H}=0 \mid \mathrm{G}=0)=0.956$ & & \\
\hline \multicolumn{2}{|c|}{ Flow OF exhaust gas } & Nonfunctional & Functional & & \\
\hline \multirow{2}{*}{ Injection OF Fluids (F) } & Nonfunctional & $P(F=1 \mid G=1)=0.979$ & $P(F=1 \mid G=0)=0.012$ & & \\
\hline & Functional & $\mathrm{P}(\mathrm{F}=0 \mid \mathrm{G}=1)=0.021$ & $\mathrm{P}(\mathrm{F}=0 \mid \mathrm{G}=0)=0.988$ & & \\
\hline \multicolumn{2}{|c|}{ Flow OF exhaust gas } & Nonfunctional & Functional & & \\
\hline \multirow{2}{*}{$\begin{array}{c}\text { Residence time OF } \\
\text { catalysis (R) }\end{array}$} & \begin{tabular}{|l|} 
Nonfunctional \\
\end{tabular} & $P(R=1 \mid G=1)=0.963$ & $P(R=1 \mid G=0)=0.027$ & & \\
\hline & Functional & $P(R=0 \mid G=1)=0.037$ & $P(R=0 \mid G=0)=0.973$ & & \\
\hline \multicolumn{2}{|c|}{ High Temperature OF elements } & \multicolumn{2}{|c|}{ Nonfunctional } & \multicolumn{2}{|c|}{ Functional } \\
\hline \multicolumn{2}{|c|}{ Residence time OF catalysis } & Nonfunctional & Functional & Nonfunctional & Functional \\
\hline \multirow{2}{*}{$\begin{array}{c}\text { Heat and mass } \\
\text { Transfer OF elements }\end{array}$} & \begin{tabular}{|l|} 
Nonfunctional \\
\end{tabular} & $P(T=1 \mid H=1, R=1)=0.993$ & $P(T=1 \mid H=1, R=0)=0.834$ & $P(T=1 \mid H=0, R=1)=0.982$ & $P(T=1 \mid H=0, R=0)=0.005$ \\
\hline & Functional & $P(T=0 \mid H=1, R=1)=0.007$ & $P(T=0 \mid H=1, R=0)=0.166$ & $P(T=0 \mid H=0, R=1)=0.018$ & $\mathrm{P}(\mathrm{T}=0 \mid \mathrm{H}=0, \mathrm{R}=0)=0.995$ \\
\hline \multicolumn{2}{|c|}{ Heat and mass transfer OF elements } & \multicolumn{2}{|c|}{ Nonfunctional } & \multicolumn{2}{|c|}{ Functional } \\
\hline \multicolumn{2}{|c|}{ Injection OF fluids } & Nonfunctional & Functional & Nonfunctional & Functional \\
\hline \multirow{2}{*}{$\begin{array}{c}\text { Amount OF } \\
\text { Contaminants }(\mathrm{C}) \\
\end{array}$} & \begin{tabular}{|l|} 
Nonfunctional \\
\end{tabular} & $P(C=1 \mid T=1, F=1)=0.998$ & $P(C=1 \mid T=1, F=0)=0.862$ & $P(C=1 \mid T=0, F=1)=0.334$ & $\mathrm{P}(\mathrm{C}=1 \mid \mathrm{T}=0, \mathrm{~F}=0)=0.059$ \\
\hline & Functional & $P(C=0 \mid T=1, F=1)=0.002$ & $P(C=0 \mid T=1, F=0)=0.138$ & $\mathrm{P}(\mathrm{C}=0 \mid \mathrm{T}=0, \mathrm{~F}=1)=0.666$ & $\mathrm{P}(\mathrm{C}=0 \mid \mathrm{T}=0, \mathrm{~F}=0)=0.941$ \\
\hline \multicolumn{2}{|c|}{ Heat and mass transfer OF elements } & Nonfunctional & Functional & & \\
\hline \multirow{2}{*}{$\begin{array}{c}\text { Backpressure AT } \\
\text { outlet (B) }\end{array}$} & Nonfunctional & $\mathrm{P}(\mathrm{B}=1 \mid \mathrm{T}=1)=0.918$ & $\mathrm{P}(\mathrm{B}=1 \mid \mathrm{T}=0)=0.069$ & & \\
\hline & Functional & $\mathrm{P}(\mathrm{B}=0 \mid \mathrm{T}=1)=0.082$ & $\mathrm{P}(\mathrm{B}=0 \mid \mathrm{T}=0)=0.931$ & & \\
\hline \multicolumn{2}{|c|}{ Backpressure AT Outlet } & \multicolumn{2}{|c|}{ Nonfunctional } & \multicolumn{2}{|c|}{ Functional } \\
\hline \multicolumn{2}{|c|}{ Amount OF Contaminants } & Nonfunctional & Functional & Nonfunctional & Functional \\
\hline \multirow{2}{*}{$\begin{array}{l}\text { Saturation OF filters } \\
\text { (S) }\end{array}$} & \begin{tabular}{|l|} 
Nonfunctional \\
\end{tabular} & $\mathrm{P}(\mathrm{S}=1 \mid \mathrm{B}=1, \mathrm{C}=1)=0.984$ & $P(S=1 \mid B=1, C=0)=0.743$ & $\mathrm{P}(\mathrm{S}=1 \mid \mathrm{B}=0, \mathrm{C}=1)=0.946$ & $P(S=1 \mid B=0, C=0)=0.022$ \\
\hline & Functional & $P(S=0 \mid B=1, C=1)=0.016$ & $P(S=0 \mid B=1, C=0)=0.257$ & $P(S=0 \mid B=0, C=1)=0.054$ & $P(S=0 \mid B=0, C=0)=0.978$ \\
\hline
\end{tabular}

Figure 9: Chart with the probabilities values (CPT) obtained for each one of the functions on the BN. 


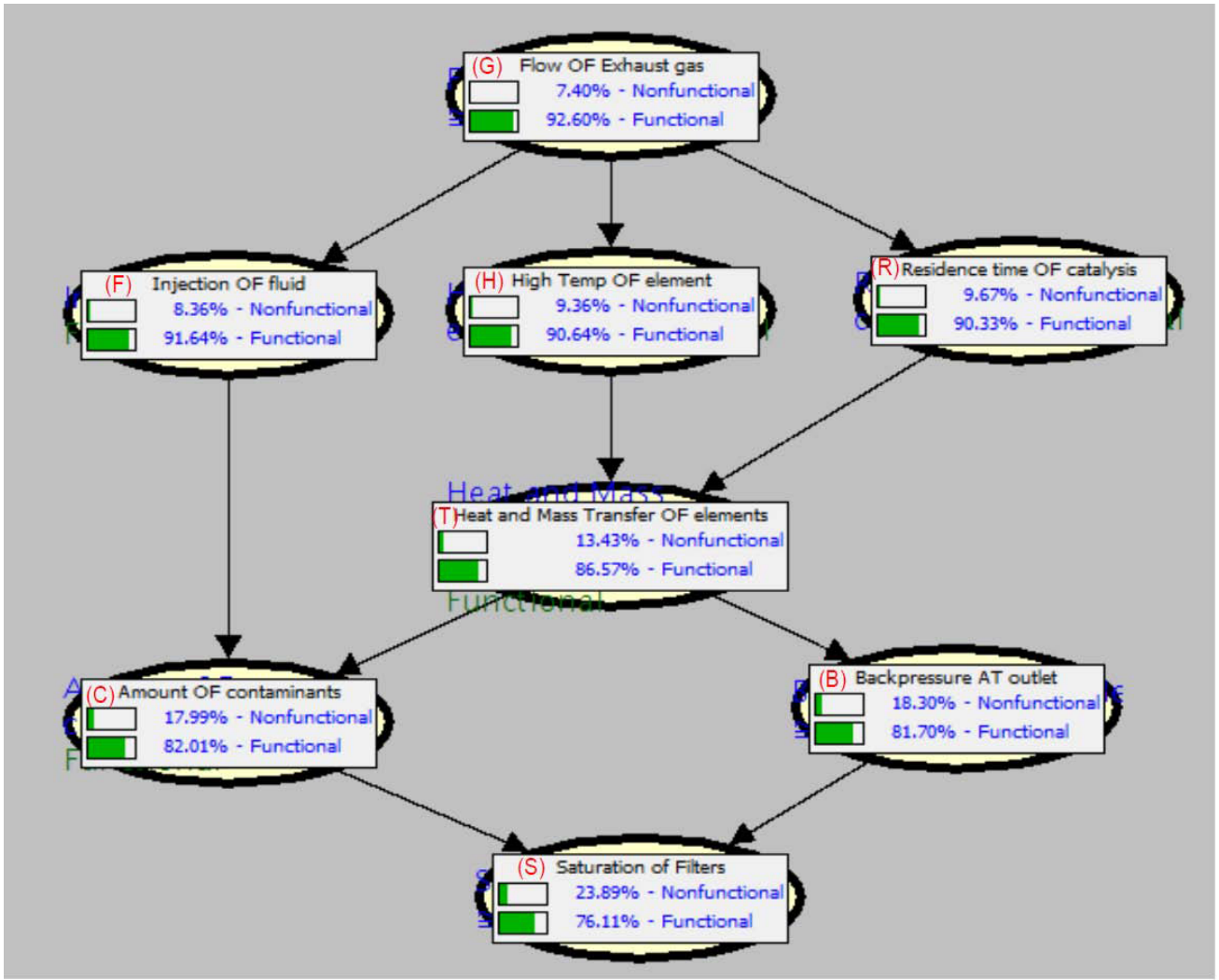

Figure 10: Bayesian Network from example on Samlam with monitors displayed

functional about $82 \%$ of the time; or $\mathrm{P}(\mathrm{C}=0)=0.82$. A sensitivity analysis was proposed for the event shown in in Equation 6.

$$
\mathrm{P}(C=1) \leq 0.1
$$

The sensitivity analysis was conducted by using the ShenoyShafer algorithm as it is one of the main methods for probability propagation in a joint tree [51]. The sensitivity analysis section of the software is depicted in Figure 11 and it presents the event constraint established in Equation 6. After running the analysis, it resulted in two different recommendations. First, Figure 11a is the multiple parameter suggestion for C's CPT where the recommended changes are highlighted in red. The second alternative is shown in Figure $11 \mathrm{~b}$ where, in a similar manner, presents the highlighted recommendations for T's CPT.

The reliability team presented the results to the design team and suggested to study both options and their the Log-odds or $\Delta l o$ [52] provided by the software. Log-odds represents the difference of the natural logarithm of the odds after applying a change in the parameters. The definition of $\Delta l o$ is stated on below:

$$
\underset{\ln \left(\mathrm{O}_{\text {Function }}=\mathrm{X} \mid \text { Pre }_{i}\right) \mid}{\Delta l o\left(\mathrm{P} \quad\left(\text { Function }_{i}=\mathrm{X} \mid \text { Pre }_{i}\right)\right)=\mid} \ln \left(\mathrm{O}^{\prime}\left(\text { Function }_{\mathrm{i}}=\mathrm{X} \mid \text { Pre }_{i}\right)\right)
$$

Where $\mathrm{X}$ is a binary variable ( 0 or 1 ) and $\mathrm{O}$ (Function $i=\mathrm{X} \mid$ Prei) represents the odds for function $\mathrm{i}$ equal to $\mathrm{X}$ given its predecessors. $\mathrm{O}\left(\right.$ Function $_{i}=\mathrm{X} \mid$ Pre $_{i}$ denotes the odds of that event after having applied the suggested change. Hence, the greater the value of $\Delta l o$, the greater the required change. In consequence, after comparing $\Delta l o(C) \approx 2.31$ versus $\Delta l o(T) \approx 5.31$, it was decided to evaluate the feasibility of the recommendations for $C$ 's CPT or option in Figure 11a. The final decision can be validated when studying the highlighted recommendations on the CPTs. For example, Figure $11 \mathrm{~b}$ is recommending that $\mathrm{P}(\mathrm{T}=0 \mid \mathrm{H}=1$, $\mathrm{R}=1)=0.007$ needed to change to $P(T=0 \mid H=1, R=1) \approx 0.658$ even further for $P(T=0 \mid H=1, R=0)=0.166$ to $P(T=0 \mid H=1, R=0) \approx 0.981$ The difference between the original and the suggested values for both probabilities is considerable high that makes the suggestion infeasible with the current technology.

In any case, the teams were able to obtain an insight into the reliability of the system and, in particular, its relationship with the function (C). Furthermore, the sensitivity analysis provided a more objective decision making process. The experts involved in this study were able to determine that a more robust approach is needed in the way $\mathrm{C}$ is affected by its predecessor nodes ( $\mathrm{F}$ and $\mathrm{T})$. After further analysis on the marginal conditional probabilities it was found that the major marginal difference was on $\mathrm{P}(\mathrm{C}=0 \mid \mathrm{T}=1, \mathrm{~F}=0)=0.138$ since the highlighted suggestion was marked to be $\mathrm{P}(\mathrm{C}=0 \mid \mathrm{T}=1, \mathrm{~F}=0) \approx 0.617$. In other words, $\mathrm{C}$ needed to be functional even when $\mathrm{T}$ was nonfunctional and $\mathrm{F}$ functional around $62 \%$ of the time. However, given the change suggestion of multiple parameters, the approach that needed to take place was more about how to improve the functionality of the three functions. In a simple way, it was interpreted as the necessity to increase the conditional independence of $\mathrm{T}$ and $\mathrm{F}$ given $\mathrm{C}$. This analysis helped designers to have a strong recommendation on robust components for those functions with the aim to meet the established requirements.

\section{Extended sensitivity analysis}

The design team acknowledged what needed to be improved based on the sensitivity analysis. They studied different design features to improve C (Amount OF contaminants) given F (Injection OF fluids) 
Citation: Sanchez LM, Inc C, Pan R (2017) Obtaining Reliability Insights during a Product's Conceptual Design Process through Bayesian Network Modeling. Ind Eng Manage 6: 224. doi:10.4172/2169-0316.1000224

\begin{tabular}{|c|c|c|c|c|c|c|c|}
\hline & & \multicolumn{3}{|l|}{ Event } & \multicolumn{3}{|c|}{ Constraint } \\
\hline & & \multicolumn{3}{|c|}{ Amount OF contaminants } & $<=-$ & 0.1 & \\
\hline & & \multicolumn{3}{|c|}{$=\operatorname{Pr}($ Nonfunctional $)=0.179858$} & & & \\
\hline \multicolumn{4}{|c|}{ Start sensitivity analysis } & \multicolumn{4}{|c|}{ Settings Iools $\square$ Constrain Two Events $\square$ Show Table Details Edit CPT } \\
\hline \multicolumn{8}{|c|}{ Multiple parameter suggestions (single CPT) } \\
\hline \multicolumn{8}{|c|}{ !" } \\
\hline Variable & Log-odds & Heat and $M . .$. & \multicolumn{3}{|c|}{ Nonfunctional } & \multicolumn{2}{|c|}{ Functional } \\
\hline Amount OF contami... & 2.310217 & Injection $0 . .$. & Nonfunctional & Functic & & Nonfunctional & Functional \\
\hline \multirow{2}{*}{ teat and Mass Tran... } & 5.611778 & Nonfunctional & 0.9980 .980206 & 0.8620 .3 & 32674 & 0.3340 .047409 & 0.0590 .006184 \\
\hline & & Functional & 0.0020 .019794 & 0.1380 .6 & 17326 & 0.6660 .952591 & 0.9410 .993816 \\
\hline \multicolumn{8}{|l|}{ \#" } \\
\hline Variable & Log-odds & High Temp ... & \multicolumn{3}{|c|}{ Nonfunctional } & \multicolumn{2}{|c|}{ Functional } \\
\hline Amount OF contami... & $|2.310217|$ & Residence t... & Nonfunctional & Func & jonal & Nonfunctional & Functional \\
\hline \multirow{2}{*}{ Heat and Mass Tran... } & . 5.611778 & Nonfunctional & 0.9930 .341423 & 0.8340 & .01803 & 0.9820 .166234 & 0.0050 .000018 \\
\hline & & Functional & 0.0070 .658577 & 0.1660 & 98197 & 0.0180 .833766 & 0.9950 .999982 \\
\hline
\end{tabular}

Figure 11: Sensitivity analysis in Samlam resulting in a) Recommendations for C's CPT and b) Recommendations for T's CPT.

\begin{tabular}{|c|c|c|c|c|c|}
\hline \multicolumn{2}{|r|}{ Heat and mass transfer OF elements } & \multicolumn{2}{|c|}{ Nonfunctional } & \multicolumn{2}{c|}{ Functional } \\
\hline & Injection OF fluids & Nonfunctional & Functional & Nonfunctional & Functional \\
\hline $\begin{array}{c}\text { Amount OF } \\
\text { Contaminants }(\mathrm{C})\end{array}$ & Nonfunctional & $\mathrm{P}(\mathrm{C}=1 \mid \mathrm{T}=1, \mathrm{~F}=1)=0.997$ & $\mathrm{P}(\mathrm{C}=1 \mid \mathrm{T}=1, \mathrm{~F}=0)=0.559$ & $\mathrm{P}(\mathrm{C}=1 \mid \mathrm{T}=0, \mathrm{~F}=1)=0.092$ & $\mathrm{P}(\mathrm{C}=1 \mid \mathrm{T}=0, \mathrm{~F}=0)=0.008$ \\
\cline { 2 - 6 } & Functional & $\mathrm{P}(\mathrm{C}=0 \mid \mathrm{T}=1, \mathrm{~F}=1)=0.003$ & $\mathrm{P}(\mathrm{C}=0 \mid \mathrm{T}=1, \mathrm{~F}=0)=0.411$ & $\mathrm{P}(\mathrm{C}=0 \mid \mathrm{T}=0, \mathrm{~F}=1)=0.908$ & $\mathrm{P}(\mathrm{C}=0 \mid \mathrm{T}=0, \mathrm{~F}=0)=0.992$ \\
\hline
\end{tabular}

Figure 12: Chart for the conditional probability table for $C$ after new design feature.

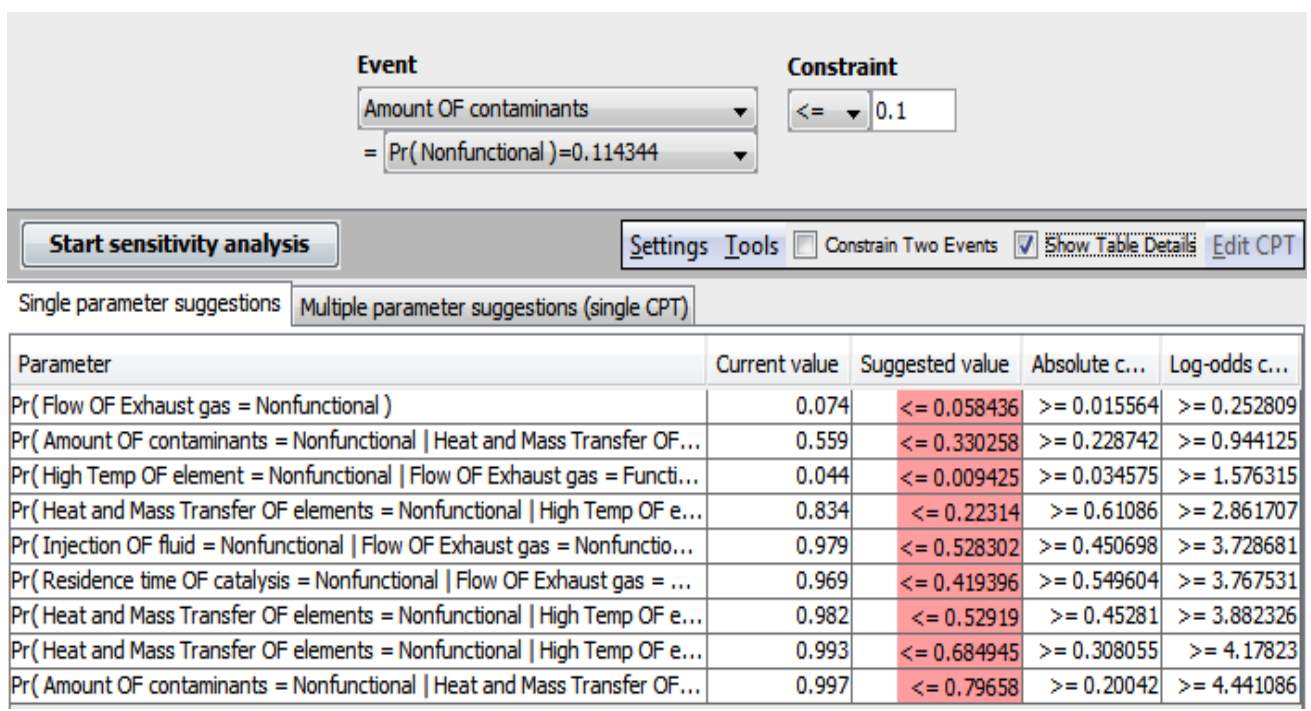

Figure 13: Second sensitivity analysis in Samlam resulting from the new design feature: sensor coating.

and the only feasible solution without impacting functionality of $\mathrm{C}$ (Amount OF contaminants) given $\mathrm{T}$ (Heat and mass transfer $\mathrm{OF}$ elements) was a new technology for sensor coating. The new coating improved $\mathrm{C}$ given the reaction it has when in contact with fluids injected while exhaust gas density and heat were not affected. Unfortunately, after an initial assessment of this new design feature, it was discovered that the suggested probabilities for C's CPT were not obtainable. The teams reunited and were provided an initial evaluation of the new coated sensor by the experts that leaded to the CPT in Figure 12.

The reliability team proposed to do a new sensitivity analysis with the new C's CPT from Figure 12. The intent of this extended sensitivity analysis was to evaluate the feasibility of the requirement in Equation 6 for other functions different of $\mathrm{C}$.

The network was updated and confirmed that $\mathrm{P}(\mathrm{C}=1) \approx 0.114$ as shown in Figure 13 which violates Equation 6. Then, also in Figure 13, the extended sensitivity analysis was run.

This analysis was able to provide single parameter suggestion as well as multiple parameter suggestions. The teams focused on the single parameter tab to evaluate the feasibility of changing a specific marginal 
conditional probability. This tab provided different opportunities for improvement. After reviewing all possible changes, it was decided to proceed with the one that involved less change or $\min \{\Delta l o\}$. Consequently, $P(G=1)=0.074$ was suggested to be change to $P(G=1)$ $\approx 0.058$. However, function $\mathrm{G}$ (Flow $\mathrm{OF}$ exhaust gas) was not under control of the design team, this function is controlled by the customer since they ensure the functionality of the "flow OF exhaust gas". Therefore, the CRD's program management team reached a warranty agreement with the customer and updated the technical profile to establish that failure rate for $G$ needed to be $P(G=1)<=0.0585$ in order for the system to meet the requirement of $\mathrm{P}(\mathrm{C}=0)>=0.9$.

\section{Evidence impact analysis}

Finally, one of the major changes that were planned for the new
CRD was regarding function B (Backpressure AT outlet). Designers needed to justify that improvements proposed to the functionality of $\mathrm{B}$ were towards having a more robust product. After consulting the reliability team it was proposed an evidence impact analysis to evaluate the system's effect on different sates of a function. Figure 14 presents the impact on the network for the two states of $\mathrm{B}$.

From this analysis, it can be appreciated graphically the different effects the states of B have on the other nodes of the network. The objective of the evidential impact analysis is to determine the positive or negative effect when evidence of a variable is available. In this case, the design team obtained the specific knowledge on the behavior of the system when $B=1$ (nonfunctional) (Figure 14b). Without the graphical representation of the system, it would be difficult to justify an improvement on $\mathrm{B}$ since the positive (or negative) impact for $\mathrm{B}=0$

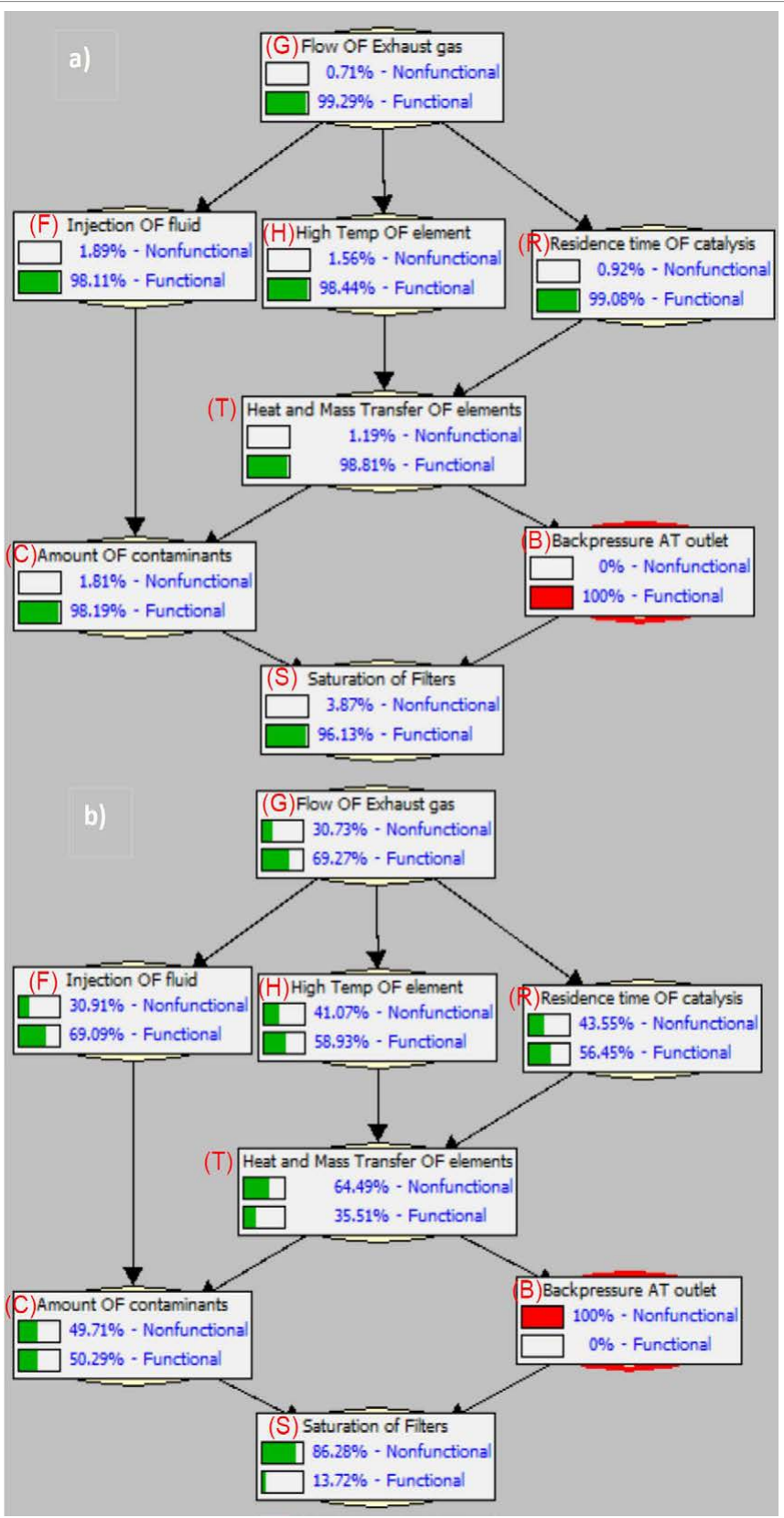

Figure 14: Bayesian network functional impact analysis of a) $P(B=0)=1$ and $b) P(B=1)=1$. 
was uncertain. On the other hand, with the $\mathrm{BN}$ as seen in Figure 14a, the improvements on the functionality of all the other nodes were quite significant. Hence, the evidence impact analysis provided the business case to improving $\mathrm{B}$ as it would deliver a more robust CRD.

\section{Discussion}

The proposed methodology can be summarized in three major steps in the conceptual design phase. The first step is functional analysis, in which the function to failure process will be defined. Once determined the functionalities, the next step is to identify and establish the relationships between functions. This task is performed by constructing a cognitive map, which formalizes those relationships in the form of a functional structure. Finally, by adding a quantitative aspect, cognitive map is transformed to a Bayesian network, with which designers have the ability to evaluate different reliability scenarios, measure functional impact of changes, or verify that product requirements are met. Thus, obtaining an insight into the reliability of a new product in its conceptual phase has been made possible by this methodology.

One of the main advantages of the proposed methodology is the graphical representation of the functional and failure structures through the $\mathrm{CM}$ and $\mathrm{BN}$. It exposes the interactions be- tween functions and facilitates the decision making process when dealing with a complex concept. Furthermore, having a reliability insight of a system in its conceptual design phase has its own advantages. For example, the necessities of some design requirements can be crossvalidated and any early design changes would be much less expensive than later changes.

The case study illustrates how the proposed methodology can serve as a general guideline for obtaining reliability insights at the conceptual design stage. It shows the utilization of $\mathrm{BN}$ through three different scenarios. In the first one, an investigation was performed to analyze how to meet the design requirement and which function needed to be improved. After the first scenario analysis, the infeasibility to meet product requirement was found, so an extended analysis was executed. The end result from the second scenario analysis conveyed the involvement of a different aspect of the reliability other than the design. It required the signed warranty agreement between customers and suppliers, which had been previously overlooked. Finally, in the third scenario the impact of changes was evaluated and the resource spending towards a more robust product was justified.

Although these scenarios only utilized the sensitivity analysis capacity of BN, the scope can be extended more broadly with different characterizations of BN. Subsequently, there are several directions to extend this study. As aforementioned, nodes with multiple states or continuous nodes can be introduced in the $\mathrm{BN}$ model and different approaches to BN quantification need to be considered for these nodes (e.g., quantum inference and genetic algorithms). Consequently, it would be worthwhile to evaluate the pros and cons of different computational tools for BN construction. Also, a functional repository could be created to expedite the design process. Finally, formulating some general guidelines for performing the robustness analysis and the simultaneous requirement analysis using $\mathrm{BN}$ will greatly benefit practitioners and help the acceptance of this methodology in the design community.

\section{References}

1. Gen M, Kim JR (1999) Ga-based reliability design: state-of-the-art survey. Computers \& industrial engineering 37: 151-155.

2. Ehrlenspiel K, Kiewert A, Lindemann U (2007) Cost-Efficient Design, SpringerVerlag Berlin Hiedelberg.
3. Fajdiga M, Jurejevcic T, Kernc J (1996) Reliability prediction in early phases of product design. Journal of Engineering Design 7: 107-128.

4. Tan C (2003) Customer-focused build-in-reliability: a case of study. International Journal of Quality \& Reliability Management 20: 378-396.

5. Hamada M, Graves T, Klaman M, Koehler A, Martz H (2007) A fully bayesian approach for combining multi-level information in multi-state fault tree quantification. Reliability Engineering and System Safety 92: 1476-1483.

6. Wang P, Kloess A, Youn BD, Xi Z (2009) Bayesian reliability analysis with evolving, insufficient, and subjective data sets. Journal of Mechanical Design 131: $259-272$.

7. Pan R, Rigdon SE (2009) Bayes inference for general repairable systems. Journal of Quality Technology 41: 82-94.

8. Johnson V, Graves T, Hamada M, Reese C (2003) A hierarchical model for estimating the reliability of complex systems. Bayesian 7: 199-213.

9. Hamada M, Martz HF, Reese CS, Graves T, Johnson V, et al. (2004) A fully bayesian approach for combining multilevel failure information in fault tree quantification and optimal follow-on resource allocation. Reliability Engineering \& System Safety 86: 297-305.

10. Wilson A, Graves T, Hamada M, Reese C (2006) Advances in data combination, analysis and collection for system reliability assessment. Statistical Science 21 514-531.

11. Pan R (2008) A bayes approach to reliability prediction utilizing data from accelerated life tests and field failure observations. Quality and Reliability Engineering International 25: 229-240.

12. Anderson-Cook CM, Graves T, Hengartner N, Klamann R, Wiedlea AC, et al. (2008) Reliability modeling using both system test and quality assurance data. Military Operations Research 13: 5-18.

13. Graves TL, Hamada MS (2010) A demonstration of modern bayesian methods for assessing system reliability with multilevel data and for allocating resources. International Journal of Quality, Statistics, and Reliability 2009: 1-10.

14. Reese CS, Wilson AG, Guo J, Hamada MS, Johnson VE (2011) A bayesian model for ntegrating multiple sources of lifetime information in system-reliability assessments. Journal of quality technology 43: 127-141.

15. Johnson V, Moosman A, Cotter P (2005) A hierarchical model for estimating the early reliability of complex systems. IEEE Transactions on Reliability 54 224-231.

16. Pahl G, Beitz W, Wallace K, Council D (2007) Engineering Design: A Systematic Approach, 3rd ed, Springer.

17. Huang Z, Jin $Y$ (2009) Extension of stress and strength interference theory for conceptual design for reliability. Journal of Mechanical Design 131: 71-81.

18. Derelov M (2008) Qualitative modelling of potential failures: on evaluation of conceptual design. Journal of engineering design 19: 201-225.

19. Bryant CR, Stone RB, McAdams DA, Kurtoglu T, Campbell MI, et al. (2005) Concept generation from the functional basis of design. In ICED 05: 15th International Conference on Engineering Design: Engineering Design and the Global Economy , Engineers Australia, pp: 280-281

20. Kurtoglu T, Tumer IY (2008) A graph-based fault identification and propagation framework for functional design of complex systems. Journal of Mechanical Design 130: 051401

21. Krus D, Grantham Lough K (2009) Function-based failure propagation for conceptual design. Artificial Intelligence for Engineering Design, Analysis and Manufacturing 23: 409-426.

22. Blanchard BS, Fabrycky WJ, Fabrycky WJ (1990) Systems engineering and analysis, vol. 4. Prentice Hall Englewood Cliffs, New Jersey.

23. Hirtz J, Stone RB, McAdams DA, Szykman S, Wood KL (2002) A functional basis for engineering design: reconciling and evolving previous efforts. Research in engineering Design 13: 65-82.

24. Van Wie M, Bryant CR, Bohm MR, McAdams DA, Stone RB (2005) A mode of function-based representations. Artificial intelligence for engineering design, analysis and manufacturing 19: 89-111.

25. Erden MS, Komoto H, van Beek TJ, DAmelio V, Echavarria E, et al. (2008) A review of function modeling: Approaches and applications. Ai Edam 22: 147-169. 
Citation: Sanchez LM, Inc C, Pan R (2017) Obtaining Reliability Insights during a Product's Conceptual Design Process through Bayesian Network Modeling. Ind Eng Manage 6: 224. doi:10.4172/2169-0316.1000224

26. Otto KN, Wood KL (2003) Product design: techniques in reverse engineering and new product development. Tsinghua University Press Ltd.

27. Stone RB, Wood KL (2000) Development of a functional basis for design. Journal of Mechanical Design 122: 359-370.

28. Stone RB, Wood KL, Crawford RH (2000) Using quantitative functional models to develop product architectures. Design Studies 21: 239-260.

29. Tumer IY, Stone RB (2003) Mapping function to failure mode during component development. Research in Engineering Design 14: 25-33.

30. Grantham Lough K, Stone R, Tumer IY (2009) The risk in early design method. Journal of Engineering Design 20: 155-173.

31. Augustine M, Yadav OP, Jain R, Rathore A (2012) Cognitive map-based system modeling for identifying interaction failure modes. Research in Engineering Design 23: 105-124.

32. Mejia Sanchez L, Pan R (2011) An enhanced parenting process: Predicting reliability in product's design phase. Quality Engineering 23: 378-387.

33. Cooke R (1991) Experts in Uncertainty: Opinion and Subjective Probability in Science. Oxford University Press.

34. Tolman EC (1948) Cognitive maps in rats and men. Psychological review 55: 189-208.

35. El-Haik B (2005) Axiomatic quality: integrating axiomatic design with six-sigma, reliability, and quality engineering. John Wiley \& Sons, p: 312.

36. Lee KC, Chung N (2006) Cognitive map-based web site design: empirical analysis approach. Online Information Review 30: 139-154.

37. Lee H, Kwon SJ (2014) Ontological semantic inference based on cognitive map. Expert Systems with Applications 41: 2981-2988.

38. Kosko B (1986) Fuzzy cognitive maps. International Journal of man-machine studies 24: 65-75.

39. Glykas M (2010) Fuzzy Cognitive Maps: Advances in Theory, Methodologies, Tools and Applications. Springer.
40. Stach W, Kurgan L, Pedrycz W, Reformat M (2005) Genetic learning of fuzzy cognitive maps. Fuzzy sets and systems 153: 371-401.

41. Ben-Gal I (2007) Bayesian networks. Encyclopedia of statistics in quality and reliability.

42. Langseth $\mathrm{H}$, Portinale $\mathrm{L}$ (2007) Bayesian networks in reliability. Reliability Engineering \& System Safety 92: 92-108.

43. Bishop CM (2006) Pattern recognition and machine learning, springer New York.

44. Stone RB, Tumer IY, Van Wie M (2005) The function-failure design method Journal of Mechanical Design 127: 397-407.

45. Nadkarni S, Shenoy PP (2001) A bayesian network approach to making inferences in causal maps. European Journal of Operational Research 128: 479-498.

46. Nadkarni S, Shenoy PP (2004) A causal mapping approach to constructing bayesian networks. Decision Support Systems 38: 259-281.

47. Aktas E, Ulengin F, Sahin SO (2007) A decision support system to improve the efficiency of resource allocation in healthcare management. Socio Economic Planning Sciences 41: 130-146.

48. Yontay P, Pan R, Mejia Sanchez L (2015) Bayesian network for reliability prediction in functional design stage. In The Proceeding of Reliability and Maintainability Symposium. IEEE.

49. Shachter RD, Peot MA (2013) Simulation approaches to general probabilistic inference on belief networks. UAl '89 Proceedings of the Fifth Annual Conference on Uncertainty in Artificial Intelligence, pp: 221-234.

50. Spiegelhalter DJ, Dawid AP, Lauritzen SL, Cowell RG (1993) Bayesian analysis in expert systems. Statistical science 8: 219-247.

51. Park J, Darwiche A (2003) Morphing the hugin and shenoyshafer architectures In Sym- bolic and Quantitative Approaches to Reasoning with Uncertainty Lecture Notes in Computer Science 2711: 149-160.

52. Chan H, Darwiche A (2001) When do numbers really matter? In Proceedings of the Seventeenth conference on Uncertainty in artificial intelligence, Morgan Kaufmann Publishers Inc. pp: 65-74. 